\title{
PREFERENCIAS ALIMENTARIAS Y POTENCIAL DISPERSOR DEL LAGARTO HERBIVORO PHYMATURUS FLAGELLIFER (TROPIDURIDAE) EN LOS ANDES
}

\section{DIET PREFERENCES AND THE HERBIVOROUS LIZARD PHYMATURUS FLAGELLIFER (TROPIDURIDAE) AS POTENCIAL SEED DISPERSER IN THE ANDES}

\author{
Constanza Celedón-Neghme ${ }^{1}$, Cristián R. Salgado² \& Pedro. F. Victoriano ${ }^{1}$ \\ ${ }^{1}$ Departamento de Zoología, Facultad de Ciencias Naturales y Oceanográficas, Universidad de Concepción, \\ Casilla 160-C, Concepción, Chile.Email: cceledon@udec.cl \\ ${ }^{2}$ Programa de Doctorado en Ciencias Biológicas Area Botánica, Facultad de Ciencias Naturales y Oceanográficas, \\ Universidad de Concepción, Casilla 160-C, Concepción, Chile.
}

\begin{abstract}
RESUMEN
La mayoría de los reptiles, especialmente los lagartos, son insectívoros u omnívoros, razón por la cual poco se sabe acerca del rol que juegan como dispersores de plantas. Phymaturus flagellifer es una lagartija andina saxícola y herbívora. De acuerdo a observaciones preliminares sobre su comportamiento y a estimaciones de su ámbito de hogar, esta lagartija liberaría semillas lejos de la planta madre, y jugaría un rol como potencial dispersor. Este trabajo tiene por objetivo determinar si existe preferencia por el consumo de las distintas especies de semillas detectadas en las fecas y evaluar la legitimidad del lagarto como dispersor. Para esto, durante marzo del 2003, se recolectaron semillas de las plantas presentes en una área rocosa del Parque Nacional Laguna del Laja y en las fecas de P. flagellifer. Para estimar el efecto de la ingesta, se realizaron ensayos de germinación y pruebas de viabilidad. Para obtener una aproximación de la sombra de semilla producida por el lagarto, se midió el tamaño de ámbito de hogar del lagarto. Se obtuvo un total de 1.298 semillas, de 445 fecas analizadas, determinándose las semillas de tres especies de plantas: Berberis empetrifolia, Rumex acetosella y Calandrinia sp. Los resultados sugieren que no existe preferencia por el consumo de $R$ acetosella y B. empetrifolia. En contraste, sí existe preferencia por consumir Calandrinia sp. Los ensayos de germinación y pruebas de viabilidad revelaron que el paso por el tracto digestivo de $P$. flagellifer afecta de manera diferencial a las semillas de las distintas especies de plantas. El porcentaje de germinación de $R$. acetosella fue significativamente mayor en el control, sin embargo la germinación B. empetrifolia y Calandrinia sp. no fue diferencial entre los tratamientos. El porcentaje de viabilidad de las semillas de B. empetrifolia provenientes del tracto digestivo fue significativamente mayor que aquél de semillas control. En cambio, las semillas de $R$. acetosella y Calandrinia sp., presentaron un porcentaje mayor de viabilidad en las semillas control. De acuerdo a estimaciones de ámbito de hogar, el área promedio en la cual $P$. flagellifer estaría alejando las semillas de la planta madre es de 20,75 $\pm 1,01 \mathrm{~m}^{2}$. Los resultados obtenidos no permiten legitimar a $P$. flagellifer como dispersor de estas plantas. Esta especie podría movilizar las semillas que consume a nivel local e influir, también localmente y a largo plazo, la estructura y diversidad de la flora presente en el área.
\end{abstract}

PALABRAS CLAVES: Lagarto andino, frugivoría, dispersión de semilla.

\section{ABSTRACT}

Most of the reptilian species, particularily lizards, are insectivorous or omnivorous. Due to this evidences the role which lizard could play in seed dispersal is largely unknown. Phymaturus flagellifer is an herbivorous, saxicolous Andean lizard. According to previous observations on its behavior and home range, this lizard could be depositing seeds far away from the parent plant. Thus, Phymaturus flagellifer could be suggested to be as a potential seed 
disperser. The objectives of this study are to determine if this lizard possesses dietary preferences among ingested sedds and to evaluate it is a legitimate seed disperser. For this, during March of 2003, seeds, plants, and faeces of Phymaturus flagellifer were collected in a rocky area at Laguna del Laja National Park. This lizard's home range was also estimated. To determine the effects of ingestion, we performed seed germination and viability tests. A total of 1298 seed was found, from 445 feces analyzed. The species Berberis empetrifolia, Rumex acetosella and Calandrinia sp., were identified. The results uncover no dietary preference for $R$. acetosella and $B$. empetrifolia. A dietary preference was shown for Calandrinia sp. Seed germination and viability tests revealed that percentage germination of $R$. acetosella was significantly greater in the control seeds. In the other two species, no significant differences were detected. The results of the viability test show significant differences among three species. Percentage viability of seeds of $B$. empetrifolia from the gut was higher than that of control seeds. In contrast, percentage viability of seeds of $R$. acetosella and Calandrinia $s p$. was lower than that of those that had passed through the digestive tract. Results of the home range analysis suggest that $P$. flagellifer could be dispersing seeds only a short distance away from the parent plant (i.e., $\left.20.75 \pm 1.01 \mathrm{~m}^{2}\right)$. Thus, P. flagellifer can not be legitimized as a seed disperser of these plants. However, this lizard could be mobilizing seeds at a local scale, thus, potentially influencing long-term local plant community structure and diversity in this area.

KEYwORDS: Andean lizard, frugivory, seed dispersal.

\section{INTRODUCCION}

La etapa de semilla constituye la única fase móvil de una planta con su material genético completo. Durante este período, las semillas son removidas y alejadas de la planta madre a través de la dispersión. Este proceso es parte de un complejo ciclo integrado por una sucesión de procesos que conectan generaciones sucesivas de plantas (Wang \& Smith 2002) y puede afectar los procesos de germinación y reclutamiento. Ambos procesos influyen sobre la disponibilidad de frutos, la distribución espacial y la estructura espacial de la siguiente generación (Wang \& Smith 2002).

Variadas modificaciones en las diásporas determinan diversos mecanismos de dispersión en plantas (Ridley 1930, Van der Pij1 1972). Las plantas que exhiben adaptaciones morfológicas especializadas tales como: estructuras adherentes, ganchos, apéndices comestibles y frutos carnosos, requieren la intervención de un animal como agente dispersor (Berg 1975, Howe 1980, Howe \& Vande Kereckhove 1981). Cualquiera de estos mecanismos permitiría que las semillas eventualmente fuesen depositadas en micro sitios que posean condiciones favorables para germinar y establecerse (Howe \& Smallwood 1982). La dispersión de las semillas mediante animales tiene lugar a través de una variedad de mecanismos como: epizoocoria (Sorensen 1986), diszoocoria (Bossema 1979) y endozoocoria (Jansen 1983). Esta última implica la ingestión de las diásporas, que son diseminadas una vez que han pasado por el tracto digestivo (TD) del animal y son expulsadas junto con las fecas.
La mayor parte de los estudios que informan acerca del potencial dispersor de aves y mamíferos, determinan que un gran número de especies de plantas sean diseminadas por estos vertebrados (Forget 1996, Julliot 1997, Forget \& Vander Wall 2001). Sin embargo durante los últimos 15 años los reptiles, especialmente los lagartos, han recibido mayor atención (Godinez-Alvarez 2004). En los estudios realizados existe evidencia del aumento en la velocidad de germinación de las semillas que fueron ingeridas por una especie de lagartija (Willson et al. 1996). Otro estudio sugiere que el paso de las semillas a través del TD de una lagartija tiene un efecto abrasivo sobre la testa, lo que facilita la entrada de agua y el intercambio gaseoso (Castilla 2000). Las semillas pueden ser retenidas en el TD por algunos días (Willson et al. 1996), aunque el tiempo de retención en el interior del lagarto parece no influir significativamente sobre el porcentaje de germinación (Castilla 2000).

Trabajos recientes revelan que los procesos de polinización y dispersión a través de lagartijas son más comunes en islas (Olesen \& Valido 2003). Varios estudios han enfatizado la importancia que podrían tener los lagartos omnívoros o herbívoros en el proceso de dispersión en este tipo de ambientes (e.g., Sadek 1981, Machado 1985, Fellers \& Drost 1991), notando que los frutos carnosos constituyen gran parte de sus dietas (Valido \& Nogales 1994). El hecho de que nuestro país sea considerado una isla biogeográfica y que las especies de lagartos se distribuyan en Chile prácticamente en todas las regiones ecológicas conocidas (Veloso et al. 1995) nos sugieren que los lagartos podrían jugar un rol 
importante en la interacción con plantas. Entre los lagartos chilenos además existen especies limitadas a la Cordillera de los Andes. En ambientes andinos no se conoce el rol dispersor de lagartijas, sin embargo se ha registrado la ingestión de frutos por parte de éstas (Fuentes 1976). En este contexto, Chile ofrece una inusual oportunidad para el estudio de la dispersión a través de lagartos en bosques templados (Willson et al. 1996). Esta región presenta una fauna dispersora de aves y mamíferos relativamente pobre (Armesto \& Rozzi 1989) y grandes radiaciones locales de taxa de lagartijas (Veloso et al. 1995).

Investigaciones previas sugieren que la dieta de los lagartos en sitios con condiciones extremas es del tipo oportunista, sin presentar preferencias por uno u otro alimento y que son ingeridos según su disponibilidad, provocando la ingesta de plantas de variadas especies y con distinta biología (Valido \& Nogales 1994, Valido et al. 2003). Por consiguiente, podría predecirse que los efectos del paso por el TD de lagartos sobre la germinación y viabilidad, en estos tipos de ecosistemas, fuese variable según el tipo de planta (Lieberman \& Lieberman 1986, Valido \& Nogales 1994, Willson et al.1996).

El lagarto Phymaturus flagellifer, saxícola, herbívoro y de distribución andino-patagónica, consume, entre otras especies, los frutos de Berberis empetrifolia (Lam.) (Berberidaceae), Rumex acetosella (Linnaeus 1753) y Calandrinia sp. (Portulacaceae), según observaciones preliminares realizadas en terreno y por la detección de semillas en las fecas. Los objetivos de este trabajo son: 1) Determinar, a través del análisis del contenido de las semillas en las fecas y de la oferta ambiental, si existe preferencia por el consumo de alguna de las plantas ingeridas por $P$. flagellifer; 2) Determinar el efecto del paso por el TD sobre el porcentaje de germinación y viabilidad de las semillas de las distintas especies ingeridas por P. flagellifer, y 3) Estimar la distribución de las semillas dispersadas alrededor de la planta madre (sombra de semilla) producida por las fecas de $P$. flagellifer; mediante la estimación de su ámbito de hogar.

\section{MATERIALES Y METODOS}

Sistema de estudio

El estudio se llevó a cabo en el Parque Nacional Laguna del Laja (37 $\left.20^{\circ} \mathrm{S} ; 71^{\circ} 18^{\prime} \mathrm{O}\right)$ (1.400 msnm). En un pequeño valle que hace aproximadamente 30 años correspondió a una bahía del lago Laja. Posteriormente, debido al descenso del nivel del agua, éste quedó expuesto, dejando una matriz de sedimentos finos sobre la cual hay abundantes rocas basálticas de diferente tamaño. En invierno el área queda totalmente cubierta por una capa de aproximadamente 2 metros de nieve. La fase de invierno riguroso comprende desde junio a septiembre. $P$. flagellifer es un lagarto herbívoro que se distribuye en Chile entre los $32^{\circ}$ y $37^{\circ}$ de latitud Sur. Este lagarto es herbívoro y coexiste con especies de Liolaemus del grupo montícola, y con el anfibio Pleurodema bufonina (Victoriano datos no publicados). La vegetación del área está dominada por especies de plantas anuales como Rumex acetosella, maleza tóxica y cosmopolita, cuyo fruto es una nuez de aproximadamente 1,3-1,5 mm de largo. Otra especie conspicua es Calandrinia sp. (Portulaceae), que posee un fruto no carnoso de tipo cápsula, de 6-9 $\mathrm{mm}$ de largo, con abundantes semillas de $1 \mathrm{~mm}$ de largo. También existen parches del arbusto Berberis empetrifolia (Berberidaceae) presente en la cordillera de la Costa y Andes hasta los $2.300 \mathrm{msnm}$ desde $30^{\circ}$ Sur hasta Magallanes. Es un arbusto achaparrado que no sobrepasa los $50 \mathrm{~cm}$ de altura y es el único que tiene frutos carnosos en el sistema. Sus frutos son bayas subglobosas de color morado oscuro, cuyas semillas miden aproxima-damente 3-4 mm y el número de semillas varía entre 3 y 7 .

\section{OBTENCIÓN DE LAS SEMILLAS}

El período de muestreo comprendió la época de actividad de los lagartos, entre los meses de septiembre 2002 y marzo 2003 (Habit \& Ortiz 1996). Las semillas control (CT) fueron recolectadas en forma directa de las plantas presentes en el área. En el caso de los frutos de $B$. empetrifolia, el pericarpio fue removido en el laboratorio. Las semillas que pasaron por el TD del animal fueron obtenidas mediante la colecta de las fecas de $P$. flagellifer con un total de 445 muestras de fecas. Estas son inconfundibles respecto a las de lagartijas sintópicas, ya que son de mayor tamaño y no presentan restos de insectos. La extracción de las semillas de las fecas se realizó en el laboratorio con la ayuda de pinzas y agua destilada. Una vez extraídas las semillas fueron contadas y determinadas a nivel de especie. La determinación fue a través de la comparación de las semillas extraídas de las plantas, con las provenientes de las fecas.

Composición de LA dieta de P. FLAGELlifer Y 
EVALUACIÓN DE PREFERENCIAS EN EL CONSUMO DE SEMILLAS

En enero de 2004 se dispuso siete transectas en el área de trabajo, de $50 \mathrm{~m}$ cada una, con el fin de determinar la abundancia relativa de las tres especies de plantas. Además se efectuó un conteo de los frutos por planta con el fin de obtener el número promedio de semillas producidas por cada individuo. A través de estos datos se obtuvo la oferta relativa de semillas en el área. El consumo relativo de los frutos de estas plantas se determinó mediante el porcentaje de aparición de las semillas de las tres especies en las fecas. Estos datos fueron ingresados al programa Krebs Ecological Methodology con el fin de determinar si existe algún tipo de preferencia por el consumo de las semillas de alguna de las tres plantas. El programa arrojó tres valores: la tasa de forrajeo es una medida simple de preferencia (Savage 1931), cuyo valor $(0 \rightarrow \infty)$ representa la razón entre el consumo y la oferta. El índice de selectividad de Manly es la versión normalizada de la tasa de forrajeo. Valores inferiores a $1 / \mathrm{m}(\mathrm{m}=$ número de especies disponibles para el consumo, en este estudio $\mathrm{m}=3$ ) indican rechazo, valores iguales indiferencia y superiores selección (Krebs 1999). El valor alfa de Manly es un índice de preferencia y se deriva de la probabilidad de encuentro del depredador con la planta y la probabilidad de captura en el encuentro (Manly et al. 1972, Chesson 1978). Valores inferiores que 1/ $\mathrm{m}$ indican rechazo, y superiores preferencia.

\section{ENSAYOS DE GERMINACIÓN}

Las semillas obtenidas de las fecas (TD) y las semillas obtenidas de las plantas (CT), fueron puestas a germinar en placas petri a $25^{\circ} \mathrm{C}$ en una cámara de germinación de acuerdo a datos de temperaturas de referencia obtenidos en terreno. Se utilizaron siempre cuatro réplicas por tratamiento. El número de semillas por réplica en el ensayo para cada una de las especies fue determinado según la abundancia y disponibilidad de las semillas en las fecas (Tabla I). El criterio para considerar una semilla germinada fue la emergencia visible de la radícula (Baskin \& Baskin 1998).

Las semillas que no germinaron durante el período de observación correspondiente a 30 días fueron sometidas a una prueba de viabilidad utilizando TTC (2, 3, 5-triphenyl-2H-tetrazolium), con el fin de determinar si el embrión estaba vivo o muerto (Moore 1973). Con el propósito de establecer si existen diferencias significativas en los porcentajes de germinación y viabilidad de las plantas entre los tratamientos, se aplicó una prueba t-Student con varianza ponderada para cada una de las especies que cumplían con los supuestos de homogeneidad de varianzas y distribución normal de los datos (Zar 1996). Para las especies que no cumplieron con estos supuestos se realizó una prueba no paramétrica de Mann-Whitney U (Zar 1996).

\section{DETERMINACIÓN DEL ÁMBITO DE HOGAR}

Tabla I. Número de semillas por replica para el tratamiento y el control.

TABLE I. Seeds number for each replicate for treatment and control.

\begin{tabular}{ccc}
\hline Especies & Control(CT) & Tracto (TD) \\
\hline Berberis empetrifolia & 10 & 10 \\
Rumex acetosella & 35 & 15 \\
Calandrinia sp. & 30 & 30 \\
\hline
\end{tabular}

Esta estimación se realizó con el fin de hacer una aproximación al área potencial de dispersión por parte de los lagartos. Para obtener las medidas de los ámbitos de hogar de cada individuo, se trazó una grilla de $25 \times 25 \mathrm{~m}$ alrededor de una roca de $5 \times$ $6 \mathrm{~m}$, donde reside un harem de P. flagellifer. Este detalle resulta crucial en este caso, ya que la finalidad era obtener una aproximación del tamaño de la sombra de semilla producida por las fecas del lagarto, a través de la determinación de su ámbito de hogar. Por consiguiente fueron tomadas las coordenadas de movimiento X e Y para cada uno de los 11 lagartos residentes. Los animales fueron marcados con series de colores con pintura acrílica para su identificación. Las series de datos con las coordenadas fueron ingresadas al programa Calhome (California Home Range) y fueron analizadas a través del método Adaptative Kernel. Se obtuvo el valor del tamaño del ámbito de hogar de cada uno de los individuos del harem. Con estos valores se calculó el promedio y el respectivo error estándar. A través de esto se obtuvo un valor promedio de la distancia a la cual $P$. flagellifer podría alejar las semillas de la planta madre. 


\section{RESULTADOS}

COMPOSICIÓN DE LA DIETA DE $P$. FLAGELLIFER Y PREFERENCIAS EN EL CONSUMO DE SEMILLAS

Un total de 1.298 semillas fueron halladas en 445 fecas. La dieta del lagarto es mayoritariamente herbívora. Las fecas sólo presentaban restos de frutos y plantas (hojas, tallos, flores, etc.), incluyendo semillas y en ocasiones restos de formícidos (Tabla II). Los lagartos mostraron una clara tendencia por el consumo de frutos, ya que $91 \%$ de las fecas presentó semillas.

Los resultados análisis de preferencia por el consumo de las semillas de diferentes plantas (Tabla III) revelan que la mayor tasa de forrajeo pertenece a Calandrinia sp. $(415,789)$, mientras que los valores en orden descendiente fueron para B. empetrifolia $(2,932)$ y $R$. acetosella $(0,375)$. El índice de selectividad arrojó que $P$. flagellifer tiene mayor predilección por el consumo de Calandrinia sp. $(0,995>1 / \mathrm{m})$ y $B$. empetrifolia $(0,491>1 / \mathrm{m})$ al contrario del valor revelado para $R$. acetosella $(-0,455<1 / \mathrm{m})$, cuyo resultado muestra un claro rechazo por su consumo. El índice alfa muestra una clara tendencia a preferir las semillas de Calandrinia sp. $(0,992>1 / \mathrm{m})$. En contraste, los resultados para $B$. empetrifolia $(0,007<1 / \mathrm{m})$ y $R$. acetosella $(0,001<1 / \mathrm{m})$ revelan que los lagartos evitan el consumo de estas plantas.
TABLA II. Composición de la dieta de P. flagellifer. $\% \mathrm{~S}$ : porcentaje del numero total de semillas.

TABLE II. Composition of the diet P. flagellifer. $\%$ S: seeds percentage of the total number of seeds.

\begin{tabular}{ll}
\hline Semillas encontradas en las fecas & $\mathrm{S} \%$ \\
\hline Rumex acetosella & 10 \\
Berberis empetrifolia & 6.9 \\
Calandrinia sp. & 13 \\
Verbascum tapsus & 6.9 \\
$\mathrm{NN}_{1}$ & 6.0 \\
$\mathrm{NN}_{2}$ & 15 \\
$\mathrm{NN}_{3}$ & 0.4 \\
$\mathrm{NN}_{4}$ & 45 \\
$\mathrm{NN}_{5}$ & 3.8 \\
$\mathrm{NN}_{6}$ & 8.0 \\
$\mathrm{NN}_{7}$ & 18 \\
Formicidae & 7.5 \\
\hline
\end{tabular}

TABLA III. Estimación de preferencias en la dieta de P. flagellifer.

TABLE III. Estimation of dietary preferences of $P$. flagellifer.

\begin{tabular}{lccc}
\hline & Berberis empetrifolia & Rumex acetosella & Calandrinia sp. \\
\hline Tasa de forrajeo & 2.932 & 0.375 & 415.789 \\
Indice de selectividad & $0.491>1 / \mathrm{m}$ & $-0.450<1 / \mathrm{m}$ & $0.995>1 / \mathrm{m}$ \\
Indice de preferencia & $0.007<1 / \mathrm{m}$ & $0.001<1 / \mathrm{m}$ & $0.992>1 / \mathrm{m}$ \\
\hline
\end{tabular}

ENSAYOS DE GERMINACIÓN

El porcentaje de germinación de las semillas de $B$. empetrifolia no difirió significativamente entre los tratamientos $(\mathrm{U}=4,00 ; \mathrm{Z}=-1,15 ; \mathrm{p}=0,24)$, siendo 5 $\%$ en las semillas del TD y $0 \%$ en las del CT (Fig. 1 A). En el caso de Calandrinia sp. el porcentaje de germinación en ambos tratamientos fue $0 \%$. En contraste, el porcentaje de germinación de las semillas de $R$. acetosella difiere significativamente entre los tratamientos $(\mathrm{t}=-8,063 ;$ d. $\mathrm{f}=6 ; \mathrm{p}=0,000)$ con $\mathrm{un} 73$ $\%$ en las semillas del CTy un $5 \%$ en las del TD(Fig. 1 B).
PRUEBAS DE VIABILIDAD

Los resultados revelaron diferencias significativas en el porcentaje de viabilidad de las semillas en las tres especies de plantas. Las semillas de $B$. empetrifolia procedentes del TD mostraron un 75 $\%$ de viabilidad y las semillas CT sólo un $13 \%(\mathrm{t}=$ $-6,933$; d.f $=6 ; p=0,000$ ) (Fig.2 A). Lo opuesto ocurrió con las otras dos especies, las semillas de Calandrinia sp. revelaron porcentajes superiores de viabilidad en las semillas del CT (70\%), en relación con el $14 \%$ de las provenientes del TD $(t=12,41$; 
d.f $=6 ; \mathrm{p}=0,000)($ Fig. 2 B). Las semillas de $R$. acetosella $(\mathrm{U}=0,00 ; \mathrm{Z}=2,30, \mathrm{p}=0,020$ ?) revelaron un $7 \%$ de viabilidad en las semillas del CT y $0 \%$ en las provenientes del TD ya que estaban vanas.
DETERMINACIÓN DEL ÁMBITO DE HOGAR

Se contabilizaron un total 11 individuos de P. flagellifer, un macho dominante, ocho hembras de su harem y dos juveniles. El promedio del ámbito de hogar considerando a todos los lagartos fue $20,75 \pm 1,01 \mathrm{~m}^{2}$.
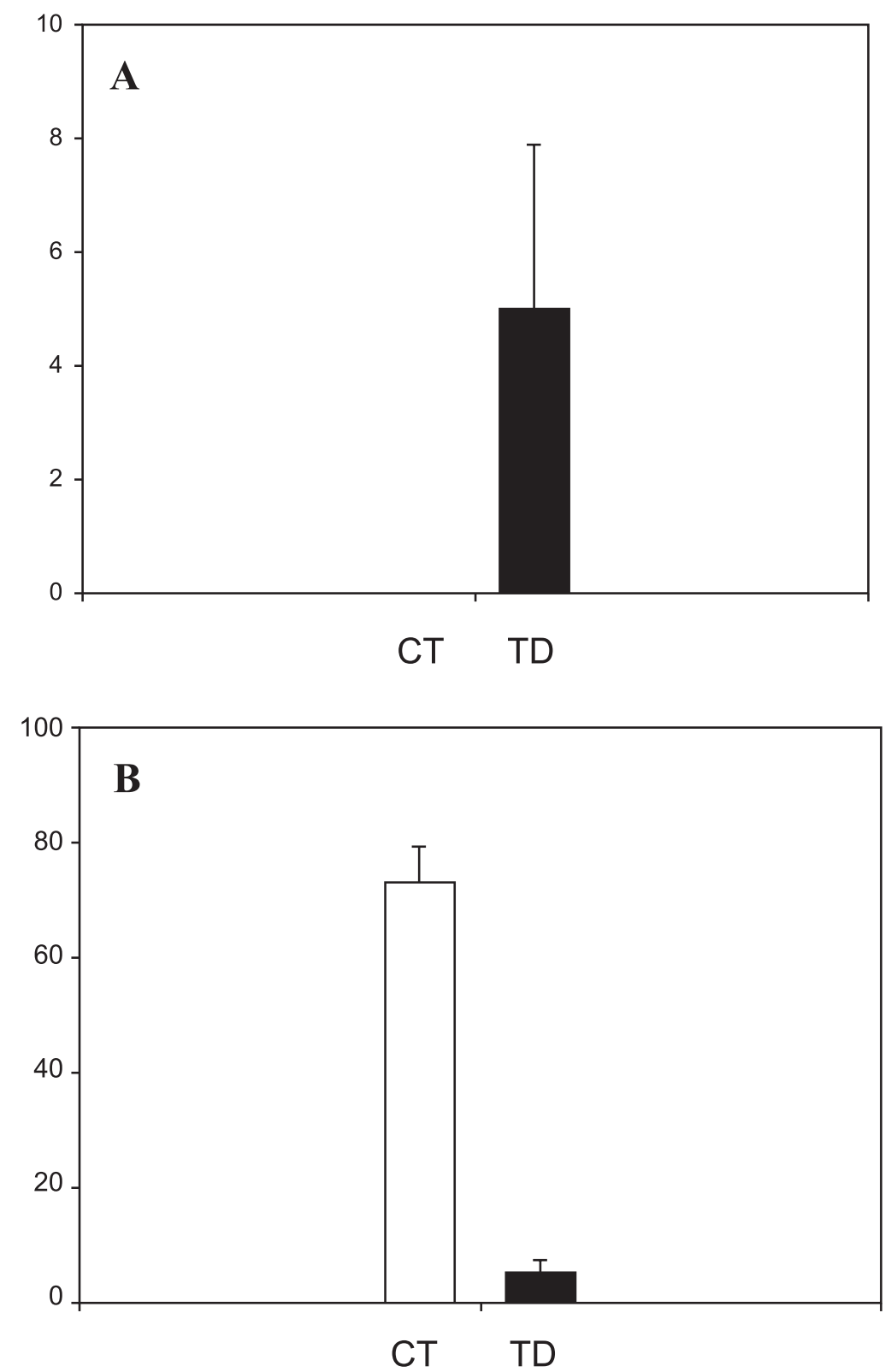

FiguRA 1. Porcentaje de germinación de B. empetrifolia (A) y porcentaje de germinación de $R$. acetosella (B).

FIGURE 1. Germination percentage in B. empetrifolia (A) and germination percentage in R. acetosella. 
Gayana 69(2), 2005
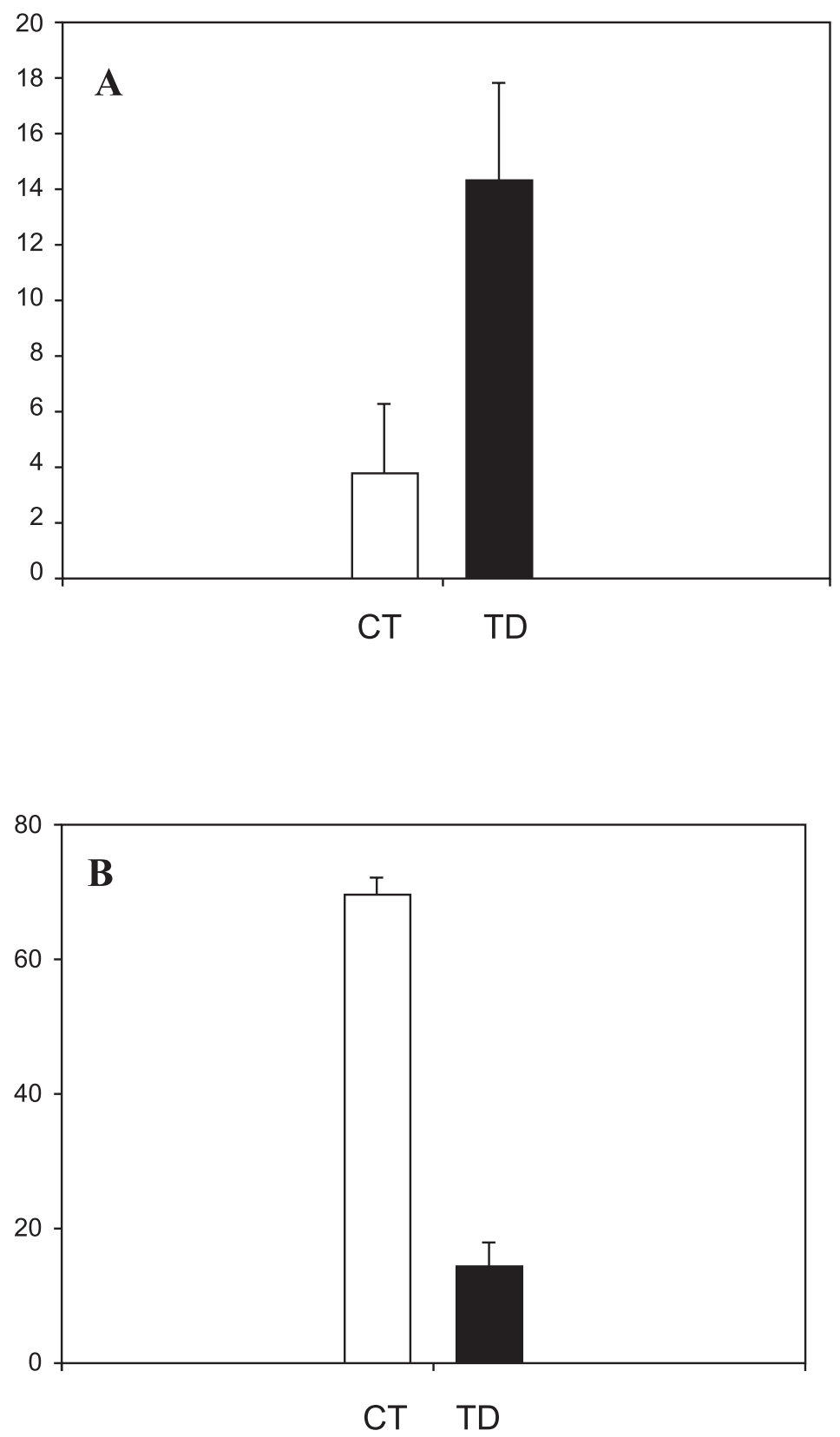

FIgURA 2. Porcentaje de viabilidad de B. empetrifolia (A) y porcentaje de viabilidad de Calandrinia sp (B). El porcentaje de viabilidad $R$. Acetosella fue cero en las semillas ingeridas por el lagarto.

Figure 2. Viability percentage in B. empetrifolia (A) and viability percentage in Calandrinia sp (B). The viability percentage for $R$. acetosella was zero in ingested seed by lizards. 


\section{DISCUSIONY YONCLUSIONES}

DIETA HERBÍVORA DE $P$. FLAGELLIFER Y PREFERENCIAS EN EL CONSUMO DE FRUTOS

El elevado porcentaje de semillas presentes en las fecas (91\%) sugiere que los lagartos muestran una clara tendencia por el consumo de frutos, además de flores y hojas. El consumo de hormigas podría ser incidental, debido a la baja frecuencia de aparición en las fecas.

Un estudio previo realizado en un lagarto de las islas Canarias mostró que no existe preferencia o evasión por el consumo de las distintas plantas (Valido \& Nogales 1994). En cambio, el presente estudio sugiere que existe un claro rechazo por el consumo de $R$ acetosella, la especie en estudio más abundante en el área $(87 \%$ ) y que además, posee un número elevado de semillas por planta $(\mathrm{X}=679)$. Esto sugiere que esta planta es poco palatable para $P$. flagellifer, lo que se puede deber al sabor que le da el ácido oxálico presente en las plantas de $R$. acetosella (Matthei et al. 1995). En cambio, de acuerdo al índice de selectividad para $B$. empetrifolia, las semillas de esta especie estarían seleccionadas. Sin embargo el valor es muy cercano al número donde la especie le es indiferente $(0,333)$. El índice alfa no muestra preferencia por el consumo de esta planta. Este resultado es paradójico, ya que se trata de una planta relativamente abundante en el área $(14 \%)$ y posee un número considerable de frutos carnosos $(X=393)$, atractivos y palatables que podrían aportar gran cantidad de energía para el consumidor. Cabría esperar que los frutos de $B$. empetrifolia fueran escogidos con mayor predilección que los frutos de las otras dos especies en estudio. Estos resultados pueden deberse a la época de muestreo y la escasez de los frutos de esta planta en el medio. Los resultados arrojados para Calandrinia sp. revelan que la tasa de forrajeo, el índice de selectividad y el índice de preferencia á muestra predilección en el consumo de esta planta. Se trata de una especie muy poco abundante en el área $(0,11 \%)$, cuyo fruto es una cápsula muy poco atractiva y bastante dura, pero con un número elevado de semillas de pequeño tamaño en su interior $(X=704)$. Este resultado puede deberse también a la época de muestreo, ya que en esta fecha el alimento escasea, y los lagartos podrían estar consumiendo lo que quede disponible.
EFECTO DEL TD SOBRE LA GERMINACIÓN Y LA VIABILIDAD El efecto que puede producir el paso de una semilla a través del TD de los vertebrados puede variar según la especie de planta (Lieberman \& Lieberman 1986). En el caso de los reptiles, algunas especies vegetales no arrojan diferencias significativas en el porcentaje de germinación entre los tratamientos (Whitaker 1987, Traveset 1990, Valido \& Nogales 1994, Willson et al. 1996, Castilla 2000). En otras especies los resultados revelan un incremento en el porcentaje de germinación de las semillas que pasaron a través del TD (Rick \& Bowman 1961, Valido \& Nogales 1994, Willson et al. 1996). En esta investigación los resultados obtenidos muestran que los ensayos de germinación realizados con las especies B. empetrifolia y Calandrinia sp. no arrojan diferencias significativas en el porcentaje de germinación. El porcentaje de germinación fue $0 \% 0$ muy cercano a este valor en ambas especies, por lo que no evidencia el efecto del paso por el TD del lagarto. Lo anterior podría deberse al hecho de que ambas especies habitan zonas que en alguna estación del año presentan una cubierta de nieve. Por lo tanto podrían requerir de un período de estratificación para romper la latencia de las semillas (Meyer et al. 1995). El período de estratificación previene la germinación precoz en las condiciones de otoño, donde los regímenes de humedad y temperatura no son los óptimos para completar la germinación de la semilla y el establecimiento de la plántula, permitiendo con esto que la germinación ocurra solo después de que la semilla ha experimentado las bajas temperaturas en invierno (Cavieres \& Arroyo 2000).

En el caso de $R$. acetosella, sí hubo diferencias significativas en el porcentaje de germinación de las semillas. Estos resultados, evidencian claramente el efecto negativo del TD sobre la germinación de las semillas de esta planta, sus extensos rizomas le permiten competir exitosamente con otras plantas (Matthei 1995). Este efecto negativo en las semillas podría resultar beneficioso para las plantas nativas del lugar (e.g. B. empetrifolia y Calandrinia sp.), pero al considerar que $R$. acetosella es poco consumida por $P$. flagellifer, el beneficio para las otras especies de plantas podría ser mínimo.

Castilla (1999), basado en observaciones sobre Podarcis lilfordi, sugirió que el porcentaje de viabilidad no difiere significativamente entre las semillas ingeridas y las del control. Este resultado 
indicaría que la ingestión de las semillas no afecta su viabilidad. No obstante en $B$. empetrifolia y Calandrinia sp. sólo fue posible evidenciar el efecto de la ingestión de sus semillas mediante la prueba de viabilidad, ya que la germinación fue casi nula en los ensayos. Las tres especies revelaron diferencias significativas en el porcentaje de viabilidad de las semillas de los distintos tratamientos. En el caso de B. empetrifolia se evidencia el efecto positivo del paso por el TD sobre la viabilidad, ya que mostró un porcentaje significativamente mayor en las semillas del TD. Las semillas del CT resultaron ser mucho más atacadas por hongos que las del TD, esto sugiere que el paso por el intestino de $P$. flagellifer podría tener un efecto esterilizador, que mataría los hongos de la testa de las semillas de esta especie. Para poner a prueba esta hipótesis se propone un proceso de esterilización y posteriores ensayos de germinación con las semillas de B. empetrifolia.

En contraste, las otras dos especies mostraron porcentajes de viabilidad menores en las semillas del TD, por lo tanto el paso por el TD resulta ser negativo sobre la viabilidad. La especie $R$. acetosella presentó una clara baja en la viabilidad de las semillas del TD. Además, al momento de efectuar los cortes para el test de viabilidad, las semillas se encontraban vanas. La testa estaba de las semillas de $R$. acetosella estaba intacta a simple vista. Los resultados parecen indicar que el proceso digestivo dentro del estómago del lagarto no sólo debilitaría la testa como se ha evidenciado por Castilla (2000). En este caso la testa sería permeada por los compuestos que realizan la digestión en el interior del lagarto y disolverían el embrión.

TAMAÑo ESTIMAdo DE USO DE HÁBITAT Y VAGILIDAD DEL LAGARTO

La utilización del hábitat de esta especie no resultó ser muy amplia, al parecer se trata de una especie de lagarto más o menos sedentario. Su comportamiento es de tipo territorial y de hábitos saxícolas. Presumiblemente por esta razón $P$. flagellifer resulta ser un dispersor local de las semillas que consume, pues su rango de movimiento es pequeño. Existen evidencias de que aves y mamíferos juegan un rol trascendental en la determinación de la estructura y diversidad de la vegetación (Dinerstein 1991). En este sentido esta especie de lagarto podría potencialmente influir, también a nivel local, y a largo plazo la estructura y diversidad de la flora presente en el área.

Debido a que el número de plantas incluidas en el estudio se limitó a tres especies, no se pudo determinar el efecto de la ingestión sobre todas las semillas consumidas por $P$. flagellifer. Sin embargo, el conocimiento parcial de la dieta nos permite decir que en el caso de $R$. acetosella el TD resulta desfavorable para la germinación y viabilidad de las semillas. Consecuentemente el lagarto no es un legítimo dispersor de esta especie a pesar de movilizar las semillas lejos de la planta madre. Con los datos obtenidos no se puede afirmar que $P$. flagellifer sea un legítimo dispersor de B. empetrifolia o Calandrinia sp. y si lo fuera dispersaría las semillas sólo a escala local, ya que no recorre grandes distancias, como lo hacen aves y mamíferos. Por otra parte, se sugiere realizar un estudio más minucioso, considerando el lugar donde el lagarto deposita las fecas. Si éstas son depositadas en un sitio desfavorable para la germinación y establecimiento de las plántulas, la movilización de las semillas resultaría incluso negativa para las plantas dispersadas. Para poder determinar si un animal es o no dispersor de un planta es necesario considerar, además del consumo de la planta, la movilización de las semillas, el efecto de la ingestión sobre la germinación, el lugar de deposición de las semillas y el reclutamiento de las plantas que fueron dispersadas; este último proceso conecta generaciones sucesivas de plantas y afecta la abundancia, disponibilidad de frutos, distribución espacial y estructura de la siguiente generación.

\section{AGRADECIMIENTOS}

Este proyecto fue parcialmente financiado por el proyecto DIUC 200.113.054-1.3 y proyecto Instrumental Científico-2001. Agradecemos la valiosa participación de Alejandro Muñoz en la corrección del escrito y los aportes de Alfredo Valido. También a Constanza Quiroz, Marcia González y Leonardo San Martín, por sus observaciones y la ayuda prestada en terreno. 


\section{BIBLIOGRAFIA}

Armesto, J.J. \& R. Rozzi. 1989. Seed dispersal in the rain forests of Chiloé: a survey of syndromes in comparison with other temperate and tropical forests. Journal of Biogeography 16: 219-226.

BASKIN C.C \& J.M. BASKIN 1998. Seeds Ecology, Biogeography, and Evolution of Dormancy and Germination, Academic Press. New York 666 pp.

BERG, R. Y.1975. Myrmeocochorus plants in Australia and their dispersal by ants. Australian Journal of. Botany 23: 475-508.

Bossema, I. 1979. Jays and Oaks. An eco-ethological study of a symbiosis. Behavior 65: 11-17

Castilla A.M. 1999. Podarcis lilfordi's from the Balearic Island as a potential disperser of rare Mediterranean plant Whitania frutescens. Acta Oecologica 20: 103-107.

Castilla A.M. 2000. Does passage time through the lizard Podarcis lilfordi's guts affect germination performance in the plant Withania frutescens? Acta Oecologica 21:119-124.

CAVIeres L.A. \& M.T.K. Arroyo. 2000. Seed germination response to cold stratification period and thermal regime in Phacelia secunda (Hydrophyllaceae). Plant Ecology 149: 1-8.

Chesson, J. 1978. Measuring preference in selective predation. Ecology 59: 211-215.

Dinerstein, E. 1991 Seed dispersal by greater one-horned rhinoceros (Rhinoceros unicornis) and the flora of Rhinoceros latrines. Mammalia 55: 355-362.

Fellers, G. M. \& C. A. Drost. 1991. Ecology of the island Night lizard, Xantusia riversiana, on Santa Barbara island, California. Herpetological Monograph 5: 28-78.

ForGET, P.M. 1996. Removal of seeds of Carapa procera (Meliaceae) by rodents and their fate in rain forest in French Guiana. Journal of Tropical Ecology 12: 751-761.

Forget, P-M. \& S.B. VANDER W ALl. 2001. Scatter hoarding rodents and marsupials: convergent evolution on diverging continents. Trends in Ecology and Evolution 16: 65-67.

FuENTES, E. R. 1976. Ecological convergence of lizard communities in Chile and California. Ecology 57: 3-17.

Godinez-Alvarez, H. 2004. Polinización y dispersión de semillas por lagartijas: una revisión. Revista Chilena de Historia Natural 77: (3) 569-577.

Habit E.M. \& J.C ORTiz. 1996. Ciclo reproductivo de Phymaturus flajellifer (Reptilia, Tropiduridae) Boletín Sociedad de Biología de Concepción. 67: 7-14.

Howe, H. F. 1980. Monkey dispersal and waste of neotropical fruit. Ecology.105: (61) 944-959.

Howe, H. F \& J. Smallwood. 1982. Ecology of seed dispersal. Annual Review in Ecology and Systematic 13: 201-228.

Howe, H.F. \& G.A Vande Kereckhove. 1981. Removal of wild nutmeg (Virola surinamensis) crops by birds. Ecology. 62: 1093-1106.
JANSEN,D.H. 1983. Dispersal of seeds by vertebrate guts. Pages232-262 In: D.J. Futuyma and M. Slatkin, (eds). Coevolution. Sinauer, Sunderland; Mass.

JuLliot, C. 1997. Impact of seed dispersal by red howler monkeys Alouatta seniculus on the seedling population in the under storey of tropical rain forest. Journal of Ecology 85: 431-440.

KreBs, C. J. 1999. Ecological methodology (Second Edition). Addison Wesley Longman, Inc. EE.UU. $620 \mathrm{pp}$.

Lieberman, M. \& D. Lieberman. 1986. An experimental study of seed ingestion and germination in a plantanimal assemblage in Ghana. Journal of Tropical Ecology 2: 113-126.

Machado, A. 1985. New data concerning the Hierro Giant Lizard and the Lizard of Salmor (Canary Islands). Boone Zoological Beitrage 36: 585-606.

Manly, B. F. J., Miller, P. \& L. M. CooK. 1972. Analyzes of a selective predation experiment. American Naturalist 106: 719-736.

Matthei, O. 1995. Las malezas que crecen el Chile. Santiago: Alfabeta Impresores. 545 pp.

Meyer, S., Kitchen, S. G. \& S. L.Carlson. 1995. Seed germination timing patterns in intermountain Penstemon (Scrophulariaceae). American Journal of Botany 82: 377-389.

Olesen, J. M \& A. VAlido. 2003. Lizards as pollinators and seed dispersers: an island phenomenon Trends in Ecology and Evolution 18: 177-181.

Rick, C. M. \& R. I. Bowman. 1961. Galapagos tomatoes and tortoises. Evolution 15: 407-417.

SADEK, R.A. 1981. The diet of the Madeiran lizard Lacerta dugesii. Zoological Journal of Linnaean Society 73: 313-341.

SAVAGE, R. E. 1931. The relation between the feeding of the herring off the east coast of England and the plankton of the surrounding waters. Fishery Investigation, Ministry of Agriculture, Food, And fisheries, Series 2 (12): 1-18.

Sorensen, E. 1986. Seed dispersal by adhesion. Annual Review in Ecology and Systematic 17: 443-463.

Traveset, A. 1990. Ctenosauria similis Gray (Iguanidae) as a seed disperser in central America deciduous forest. American Midland Naturalist 123: 402-404.

Valido, A. \& M. Nogales. 1994. Frugivory and seeds dispersal by the lizard Gallotia galloti (Lacertidae) in a xeric habitat of the canary islands. Oikos 70: 403-411.

Valido A., Nogales M. \& F. M. Medina. 2003. Fleshy fruits in the diet of Canarian lizards Gallotia galloti (Lacertidae) in a zerichabitat of the island of Tenerife. Journal of Herpetology 37 (4): 741747.

VAN DER PIJL, L. 1972. Principles of Dispersal in Higher Plants (2nd ed). Berlin: Springer. $162 \mathrm{pp}$.

Veloso, A., Ortiz. J. C., Navarro. J., Nuñez. H., Espejo. P. \& M. A. Labra. 1995. Reptiles. En: Diversidad biológica de Chile (eds. Simonetti, J., M. Arroyo, A. Spotorno \& E. Lozada), 450 pp. Conicyt, Santiago de Chile. 
Gayana 69(2): 277-284, 2005

WANG, B. J \& T.B. Smith. 2002. Closing the seed dispersal loop. Trends in Ecology and Evolution 17: 379-385.

Whitaker, A.H. 1987. The role of lizards in New Zealand plant reproductive strategies. New Zealand Journal of Botany 25: 315-328.
Willson, M.F., Sabag C., Figueroa J., Armesto J.J. \& M. CAVIEDES. 1996. Seed dispersal by lizard in chilean rainforest. Revista Chilena de Historia Natural 69: 339-342.

Zar, J. H. 1996. Bioestatistical analiysis,(3rd edn). Prentice may, New Jersey.

Fecha de recepción: 18/04/05

Fecha de aceptación: 15/06/05 